\title{
Maternal and neonatal outcomes in pregnant women with autoimmune diseases in Pavia, Italy
}

\author{
Iolanda Mazzucchelli ${ }^{1,2^{*}+} \mathbb{B}$, Lidia Decembrino ${ }^{1 \dagger}$, Francesca Garofoli', Giulia Ruffinazzi', Véronique Ramoni ${ }^{3}$, \\ Mariaeva Romano ${ }^{3}$, Elena Prisco ${ }^{4}$, Elena Locatelli ${ }^{4}$, Chiara Cavagnolii, Margherita Simonetta ${ }^{4}$, Annalisa De Silvestri ${ }^{5}$, \\ Piermichele Paolillo ${ }^{6}$, Arsenio Spinillo ${ }^{4}$ and Mauro Stronati ${ }^{1}$
}

\begin{abstract}
Background: The increased number of childbearing women with autoimmune diseases leads to a growing interest in studying relationship among maternal disease, therapy, pregnancy and off-spring. The aim of this study was to determine the impact of autoimmune disease on pregnancy and on neonatal outcome, taking into account the maternal treatment and the transplacental autoantibodies passage.

Methods: We studied 70 infants born to 70 pregnant women with autoimmune disease attended in Fondazione IRCCS Policlinico San Matteo, Pavia, Italy from June 2005 to June 2012. Maternal and neonatal characteristics were collected and relevant clinical, laboratory, therapeutics, sonographic and electrocardiographic investigations were recorded and analyzed.

Results: We observed a high rate of spontaneous abortions in medical history, $29 \%$, and $18.6 \%$ of preterm births and $22.9 \%$ of low birth weight $(<2500 \mathrm{~g})$. Transplacental autoantibodies passage wasn't related to maternal or obstetrical complication, but anti-Ro/SSA positive pregnancies correlated with abnormal fetal heart rate $(P=0.01)$. Pregnant women on therapy showed an higher incidence of maternal $(p=0.002)$, obstetric $(p=0.007)$ complications and an increased rate of intrauterine growth restriction $(p=0.01)$ than the untreated ones.

Conclusions: Autoimmune diseases in pregnancy require to be carefully monitored to ensure the best possible management of mothers, fetuses and newborns due to the high rate of morbidity specially in case of maternal polytherapy and/or anti-Ro/SSA positivity.
\end{abstract}

Keywords: Autoimmune disease, Pregnancy, Maternal and neonatal outcome

\section{Background}

Autoimmune diseases are a group of heterogeneous disorders characterized by the attack of the immune system against its own cells, tissue or organs. In this context the resultant autoinflammatory reaction could be antibodies mediated or cytokines/protein mediated, with consequently multiple clinical and laboratory aspects [1]. Diseases are more frequent in female gender than in male

\footnotetext{
* Correspondence: iolanda.mazzucchelli@unipv.it

${ }^{\dagger}$ Equal contributors

${ }^{1}$ Neonatal Unit and Neonatal Intensive Care Unit, IRCCS San Matteo Hospital Foundation, Pavia, Italy

${ }^{2}$ Department of Internal Medicine and Therapeutics, University of Pavia,

Pavia, Italy

Full list of author information is available at the end of the article
}

(78 \%) and they could become critical during pregnancy when the immune system is actively involved to tolerate a successful gestation process [2, 3]. A pregnant woman with an autoimmune disease, should be considered high risk because of the modulation of inflammatory process, the possible effects of therapy on the fetus and the transplacental passage of autoantibodies [4, 5]. High frequency of gestational and perinatal complications such as preeclampsia, stillbirth, spontaneous abortions (11$24 \%)$, preterm birth (13.9\%) and intrauterine growth restrictions (IUGR) (2.3\%) have been reported [6, 7]. Immunoglobulin $\mathrm{G}$ isotype antibodies can freely cross the placenta with a linear relationship between gestational age and placental transfer [5]. Literature data 
report that anti-Ro/SSA and anti-La/SSB antibodies are associated with neonatal lupus erythematosus and other associated abnormalities such as congenital heart block (CHB), cardiomyopathy, cutaneous lupus lesions, hepatobiliary disease, and hematologic cytopenias [8, 9]. Furthermore, an important issue concerns the use of drugs to control maternal symptoms and exacerbations: these substances and their metabolites can cross the placental barrier and enter into the fetal circulation; this could influence the pregnancy progression, the fetus state and the perinatal outcomes [4].

The aim of this study was to expose our experience about the impact of autoimmune diseases on pregnancy, fetus and neonate outcome, taking into account the transplacental autoantibodies passage and the maternal therapy.

\section{Methods}

This retrospective study was performed at Fondazione IRCCS Policlinico San Matteo, Pavia, Italy from June 2005 to June 2012 and data of 70 women with a diagnosed autoimmune disease and of their 70 babies were collected and analyzed. The hospital, located in the north of Italy, is characterized by clinical, teaching and research mission. All the women gave written informed consent and the data was collected in a database and analyzed as anonymous data for research purpose.

The study was approved by the Ethics Committee, IRCCS Fondazione Policlinico San Matteo, Pavia, Italy.

All the enrolled women had a diagnosed autoimmune diseases in our Division of Rheumatology according to widely used criteria [10] and had a positive test for at least one autoantibody. Exclusion criteria were: multiple pregnancy, congenital infections, perinatal asphyxia, chromosomal syndromes. Any abnormalities occurring during pregnancy were registered as well as obstetrical and maternal information. Blood tests to evaluate maternal autoantibodies were performed during pregnancy according to the clinical protocol used in our Department.

Neonatal gestational age, weight, length, head circumference, Apgar score and cord $\mathrm{pH}$ were collected at birth and adjusted for gestational and chronological age. Maternal and neonatal antinuclear autoantibodies (ANA) were tested by a standard indirect immunofluorescence technique, while anti-extractable nuclear antigen antibodies (ENA) were evaluated by commercially available ELISA kits. Cardiac and cerebral ultrasound scans were performed in the neonate at birth, while electrocardiogram (ECG) testing was done within the first week of life. Electrocardiographic parameters were evaluated with particular attention to PR interval (nv $<140 \mathrm{~ms}$ ), heart rate, QT interval corrected according to the Bazett's formula (QTc) (nv <440 ms): slightly extended if the value is in the range of $440-470 \mathrm{~ms}$ and frankly abnormal if $>470 \mathrm{~ms}$.

\section{Statistical analysis}

The Shapiro-Wilk test was used to test univariate normality of quantitative variables. When these were normally distributed, results were expressed as mean values and $\mathrm{SD}$, otherwise median values and interquartile range were used (IQR; $25-75^{\text {th }}$ percentile). Data were analyzed adequately by Student $t$ test or Mann-Whitney and by analysis of variance (ANOVA) or Kruskal-Wallis test if more than two groups were involved. All tests were twosided. $P$ values $\leq 0.05$ were considered statistically significant. Association between quantitative variables was evaluated by Spearman non parametric correlation. Qualitative variables were described by counts and percentages and data were analyzed by Fisher's exact test. Data analysis was performed using STATA statistical package (version 12; Stata Corporation, College Station, 2011, Texas, USA).

\section{Results}

Characteristics of the enrolled women are reported in Table 1 . Obstetrical history of 70 women revealed an incidence of $29 \%$ of miscarriages (40 out of 136 pregnancies).

Maternal complications observed among the 70 enrolled pregnant women were observed in 13 patients

Table 1 Characteristics of pregnant women

\begin{tabular}{ll}
\hline $\begin{array}{l}\text { Mothers, number } \\
\text { Age, mean (SD) }\end{array}$ & 70 \\
\hline Pregnancy, number (\%) & 33.3 years (5.1) \\
$\quad$ Term delivery ( $\geq 37$ weeks) & $57(81.4)$ \\
$\quad$ Preterm delivery (<37 weeks) & $13(18.6)$ \\
Mode of delivery, number (\%) & $33(47.1)$ \\
$\quad$ Vaginal delivery & $37(52.9)$ \\
Caesarean section & \\
Autoimmune diagnosed disease, number (\%): & $8(11.4)$ \\
$\quad$ Sjögren's syndrome (SS) & $9(12.9)$ \\
Systemic lupus erithematosus (SLE) & $3(4.3)$ \\
Rheumatoid arthritis (RA) & $5(7.1)$ \\
Antiphospholipid syndrome (PAPS) & $19(27.1)$ \\
Undifferentiated connective tissue disease (UCTD) $^{\text {Others }}{ }^{\mathrm{a}}$ & $8(11.4)$ \\
Not clinical manifest disease & $18(25.7)$ \\
Drug therapy during pregnancy, number (\%) & $37(52.9)$ \\
\hline
\end{tabular}

${ }^{a} n=1$ autoimmune thyroiditis, $n=4$ thrombocytopenia, $n=1$ thyroiditis and autoimmune gastritis, $n=1$ ulcerative colitis, $n=1$ Wegener's granulomatosis. All were ANA positive

bomen positive to autoantibodies without clinical features of autoimmune disease 
(19\%): 2 cases of gestational diabetes, 5 of hypertension, 1 of massive pulmonary embolism, 1 of hypertransaminasemia and hyperbilirubinemia, 1 of anemia and furthermore, 1 autoimmune uveitis, 1 onset of Systemic lupus erithematosus (SLE), 1 intrahepatic cholestasis due to the worsening of the autoimmune disease.

Obstetric complications observed among the 70 enrolled pregnant women were observed in 29 cases (41.4\%): abnormal fetal heart rate $n=7$ (10\%), meconium stained amniotic fluid $n=8$ (10.7 \%), oligohydramnios $n=3(4.3 \%)$, polyhydramnios $n=2(2.9 \%)$, threat of preterm delivery $n=3(4.3 \%)$, IUGR $n=4(5,71 \%)$, positive indirect Coombs test $n=2(2.9 \%)$.

Neonatal characteristics are reported in Table 2.

All the newborns were tested for maternal autoantibodies at birth to assess the transplacental passage (Table 3) and we didn't observe significant differences comparing data obtained from infants positive to maternal autoantibodies (46 out of 70) vs those that were negative (18 out of 70). No differences in obstetric and perinatal complications (Table 4), mode of delivery, gestational age, anthropometric parameters at birth and laboratory data, except for total bilirubin levels (7.2 vs $9.8 \mathrm{mg} / \mathrm{dL}, p=0.05$ ) were observed between infants positive to maternal autoantibodies and those negative. In regard to the tested electrocardiographic values, no

Table 2 Characteristics of infants enrolled in the study

\begin{tabular}{|c|c|}
\hline Gestational age, mean (SD) & 38 weeks (3) \\
\hline \multicolumn{2}{|l|}{ Sex, number (\%) } \\
\hline Male & $35(50.0)$ \\
\hline Female & $35(50.0)$ \\
\hline Apgar score at 1' and 5', mean (SD) & $9.0(1.5)$ and $9.7(0.9)$ \\
\hline Cord pH, mean (SD) & $7.27(0.10)$ \\
\hline \multicolumn{2}{|l|}{ Blood parameters, } \\
\hline White Blood Cells x $10^{3} / \mu$, mean ( \pm SD) & $15.3(5.9)$ \\
\hline Neutrophils $\times 10^{3} / \mu \mathrm{l}$, mean $( \pm \mathrm{SD}$ ) & $8.6(4.5)$ \\
\hline Hemoglobin g/dL, mean ( \pm SD) & $17.2(2.0)$ \\
\hline Hematocrit, \% & $51.6(6.5)$ \\
\hline Platelets $\times 10^{3} / \mu \mathrm{l}$, mean $( \pm \mathrm{SD})$ & $270.9(75.5)$ \\
\hline \multicolumn{2}{|l|}{ Anthropometric data, } \\
\hline Weight at birth, mean (SD) & 3045 gr (699) \\
\hline LBW ${ }^{a}$ number (\%) & $14(20.0)$ \\
\hline VLBW ${ }^{b}$ number (\%) & $1(1.4)$ \\
\hline ELBWc number (\%) & $1(1.4)$ \\
\hline Length, mean (SD) & $49 \mathrm{~cm}(3.5)$ \\
\hline Head circumference, mean (SD) & $34 \mathrm{~cm}(2.4)$ \\
\hline IUGR ${ }^{d}$, number $(\%)$ & $4(5.7)$ \\
\hline
\end{tabular}

aLow Body Weight (Birth weight $<2500 \mathrm{~g}$ )

${ }^{b}$ Very Low Body Weight (Birth weight $<1500 \mathrm{~g}$ )

'Extremely Low Body Weight (Birth weight $<1000 \mathrm{~g}$ )

${ }^{\mathrm{d}}$ IUGR Intrauterine growth restriction
Table 3 Autoantibodies detected in mothers and in the newborns at birth

\begin{tabular}{llll}
\hline Autoantibodies & Mothers $(n=70)$ & Infants $(n=70)$ & Placental transfer $(\%)$ \\
\hline ANA & 63 & 46 & 73 \\
anti-SSA/Ro & 22 & 22 & 100 \\
anti-SSB/La $^{\text {Others }}{ }^{\mathrm{a}}$ & 6 & 6 & 100 \\
\hline
\end{tabular}

other autoantibodies

differences were found in the two groups. We observed that Ro/SSA and La/SSB autoantibodies crossed the placental barrier in $100 \%$ of cases (Table 3 ) and five out seven neonates with abnormal fetal heart rate were born to mothers positive for anti-Ro/SSA $(p=0.01)$, of these five neonates one was born to mothers treated with hydroxychloroquine, one to mother with steroid and three to mothers without therapy. Moreover, the routine analysis of neonatal laboratory parameters of infants born to mothers positive to anti-Ro/SSA versus infants born to negative ones, didn't show any statistically difference. We noted higher hemoglobin $(p=0.007)$, higher hematocrit $(p=0.02)$ and higher total bilirubin levels $(p$ $=0.008$ ) in the off-spring of negative mothers to antiRo/SSA, all these values were however in normal ranges.

Moreover, maternal and neonatal ANA titre were highly correlated as evaluated by the Spearman rho 0.74 , $p<0.001$.

Thirty-seven (52.0\%) pregnant women received medical therapy, they were treated with one or more drugs for their autoimmune disease: 23 received steroid therapy (prednisone), 9 received hydroxychloroquine, 17 received low dose aspirin and 7 low molecular weight heparin. Statistical analysis showed a higher incidence of obstetrical $(p=0.007)$ and maternal $(p=0.002)$ complications in treated women than in non-treated ones, related to a higher incidence of preterm delivery $(p=0.06)$

Table 4 Obstetric and perinatal events compared to autoantibodies transplacental transfer

\begin{tabular}{llll}
\hline Obstetrical/perinatal events & \multicolumn{4}{l}{ Autoantibodies placental transfer } \\
\cline { 2 - 4 } & Yes $(n=46)$ & No $(n=18)$ & P-value \\
\hline Abnormal fetal heart rate & 6 & 1 & NS \\
Meconium stained amniotic fluid & 5 & 3 & NS \\
Oligohydramnios & 1 & 2 & NS \\
Polyhydramnios & 2 & 0 & NS \\
Threat of preterm delivery & 1 & 2 & NS \\
IUGR & 3 & 1 & NS \\
Positive indirect Coombs test & 2 & 0 & NS \\
Ventricular septal defect & 1 & 0 & NS \\
Cerebral alteration & 4 & 4 & NS \\
\hline
\end{tabular}

NS Not-Significant 
with low birth anthropometric parameters: weight $(p=$ $0.003)$, length $(p=0.01)$ and head circumference $(p=$ 0.004). In women undergoing both immunosuppressive and anti-coagulant/anti-platelet therapy a significant correlation with a higher incidence of IUGR newborns compared to untreated women $(p=0.01)$ was registered.

Echocardiography revealed one case of ventricular septal defect in a IUGR preterm neonate.

Cerebral ultrasound scans revealed in 8 newborns the following significant alterations: periventricular hyperechogenicity $(n=6)$, intraventricular hemorrhage which resulted in pseudocysts or in multicystic leukomalacia $(n=4)$, white matter leukodystrophy associated with agenesis of the corpus callosum $(n=2)$. The statistical analysis showed that these cases correlate significantly with mother's drug therapy, $p=0.005$, but no correlation was observed with the presence of anti-Ro/SSA, $p=0.4$.

\section{Discussion}

Our observations, in agreement with data reported by other authors, confirmed a higher frequency of miscarriages, of premature births and of low birth weight infants born to women with autoimmune disease compared to epidemiologic data of general obstetric population in developed countries [11]. Moreover, we confirmed previous literature data showing that the placental barrier is crossed by antibodies: more than $70 \%$ of ANA and $100 \%$ of anti-Ro/SSA and anti-La/ SSB $[12,13]$.

The association of anti-Ro/SSA maternal positivity with neonatal lupus complicated by the congenital sinus bradycardia, first degree AV block or elongated QTc has been reported [14]. Our findings, instead, did not reveal any neonatal lupus disease in the off-spring from positive mothers. Nevertheless, a statistically significant correlation between anti-Ro/SSA positive pregnancies and abnormal fetal heart rate at the tococardiographic tracing was detected $(p=0.01)$. The arrhythmogenic role of anti-Ro/SSA and anti-La/SSB was established in animal and in human $[13,15]$ this effect could promote CHB in newborns of mothers Ro/SSA and/or La/SSA positive. We therefore supposed that the CHB has possibly been prevented by an adequate and carefully monitored pharmacological treatment during pregnancy. In our study, in fact, $73 \%$ of the mothers anti-Ro/SSA positive received a pharmacological treatment to control their autoimmune disease.

To date, even if there is no full agreement about the effects of immunosuppressive drugs on pregnancy and on fetus/infant, high-dose of immunosuppressive treatment administered to transplanted pregnant women has been associated with a transient impairment of the immune system of the newborn, increasing the risk of neonatal infections in the first months of life [16]. Other authors carried out a study to evaluate relevant effects in newborns and children born to women receiving immunosuppressants for their autoimmune disorders. No relevant effect on neonatal immune function, as evaluated by complete blood count with differential white blood cell count, immunoglobulin concentrations, lymphocyte subpopulations, and later in response to hepatitis $B$ vaccination were revealed [17]. In our study, as well, neither neutropenia nor difference between white blood cells and neutrophils count were reported in infants born to treated women, compared to those born to untreated mothers.

Furthermore, our findings revealed a statistical significant higher incidence of obstetric and maternal complication in treated women compared to those untreated. Previously, an increased risk of preterm delivery, low birth weight and IUGR associated with immunosuppressant therapy was reported by other authors [18].

We observed significantly lower values of neonatal anthropometric parameters at birth (weight, height and head circumference) in the infants born to treated mothers compared to untreated ones. Nevertheless we have to remark that these infants had an earlier gestational age at birth too, which may be the reason of the lower anthropometric measures. Interestingly, a higher number of IUGR newborns $(p=0.01)$ was observed in the group of women receiving polytherapy than in the group of the untreated women. Unfortunately, we aren't able to conclusively address the correlation of this event either with the polytherapy or with the worsening disease, requiring itself a more important therapy to control increasing symptoms. In fact both the conditions coexist and are strictly linked.

Finally, the ultrasound evaluation showed cerebral alterations in 8 infants born to treated mothers, but these alterations didn't correlate to the mother's positivity to anti-Ro/SSA. Because the use of the drugs may be an indirect sign of the disease, it is unclear if the alterations may be related to drug therapy, to the autoimmune disease or the results of the add up of these factors. These data comply with the observations of Motta et al. [19] that suggested no specific perinatal risk factor, but a multi-factorial aetiology of brain abnormalities observed in infants born to mothers with autoimmune disease.

A recent published review highlighted that the principal immunomodulant/immunosuppressive drugs administered during pregnancy have been shown to be relatively safe, but the results are not conclusive [20]. Moreover, controlled clinical trials in pregnancy are not ethical, therefore monitoring the pregnancy of these patients is the only available tool to have safety assessment. In agreement with the cited authors we also hope that international registries of the potential drug side effects or of any useful information related to the pregnancy 
and the outcome of the off-spring will become common and easily accessible over different countries [21]. Furthermore, it must be considered that autoimmune diseases could be undiagnosed before pregnancy because the mild clinical illness that could be modified by the occurring pregnancy that interferes with the immune system. The administration of a screening questionnaire in the first trimester of gestation has shown to be a useful tool to identify in advance these subjects that could be monitored carefully and, if necessary, managed the appropriate therapy to proceed successfully with the pregnancy [22].

We have therefore to take care of this dichotomous aspect: monitoring and balancing the necessity to identify and treat maternal disease and to preserve fetal health. In fact, it is important to protect mother and fetus from the hazard of increasing symptoms without, on the other hand, compromising the newborn well-being with possible drug side effects.

We are aware of the limits of our study. The sample size didn't allow to create subgroups related to different autoimmune diseases. Furthermore, we could not state if the findings were related to maternal disease or to drugs therapy or to others causative factors, such as genetic features or neonatal independent circumstance (prematurity), because some of these conditions are very often concomitant. Finally, we had not the opportunity to follow the infants' growth, therefore we have no information about the possible sequelae afterward in childhood.

\section{Conclusion}

The take home message of this investigation is that pregnancy of mothers suffering for an autoimmune disease has to be carefully monitored. Placental transfer of maternal autoantibodies, and the possible effects of maternal therapy on the fetus/neonate are risk factors that require a careful multidisciplinary management (gynecologists, rheumatologists and neonatologists) to ensure the best possible maternal and neonatal outcome International registries describing the pattern of pregnancy and the outcome of the off-spring could be an useful tool to ameliorate the management of the mother/infant dyad.

\section{Abbreviations \\ ANA: Antinuclear autoantibodies; CHB: Congenital heart block; \\ ECG: Electrocardiogram; ENA: Anti-extractable nuclear antigen antibodies; IQR: Interquartile range; IUGR: Intrauterine growth restrictions; SLE: Systemic lupus erithematosus.}

\section{Competing interests}

The authors declare that they have no competing interests.

\section{Authors' contributions}

IM coordinated the study and data collection, wrote and submitted the manuscript. LD coordinated the study, took care of the neonates, wrote and submitted the manuscript. FG managed literature data, critically discussed the manuscript draft and revisited the final version of the manuscript. GR took care of the neonates, collect patients' consent and approved the final version of the manuscript. VR took care of autoimmune disease in pregnant women, registered data and approved the final version of the manuscript. MR took care of autoimmune disease in pregnant women, registered data and approved the final version of the manuscript. EP took care of autoimmune disease in pregnant women, registered data and approved the final version of the manuscript. EL took care of pregnancy of studied women, registered data and approved the final version of the manuscript. CC took care of pregnancy of studied women, registered data and approved the final version of the manuscript. MS took care of pregnancy of studied women, registered their data and approved the final version of the manuscript. AdS made the statistical analysis, contributed to data interpretation and approved the final version of the manuscript. PP contributed to the conception of the study, interpretation of data and approved the final version of the manuscript. AS contributed to the conception of the study, interpretation of data and approved the final version of the manuscript. MS contributed to the conception of the study, interpretation of data and critically revisited the manuscript.

\section{Acknowledgments}

The authors thank Micol Angelini and Claudia Cova, Neonatal and Neonatal Intensive Care Unit, Fondazione IRCCS Policlinico San Matteo, Pavia, Italy, for their precious support to organize the database.

\section{Author details}

${ }^{1}$ Neonatal Unit and Neonatal Intensive Care Unit, IRCCS San Matteo Hospital Foundation, Pavia, Italy. ${ }^{2}$ Department of Internal Medicine and Therapeutics, University of Pavia, Pavia, Italy. ${ }^{3}$ Division of Rheumatology, IRCCS San Matteo Hospital Foundation, University of Pavia, Pavia, Italy. ${ }^{4}$ Department of Obstetrics and Gynecology, IRCCS San Matteo Hospital Foundation, University of Pavia, Pavia, Italy. ${ }^{5}$ Biometry Unit, San Matteo Hospital Foundation, P.le Golgi 19, Pavia 27100, Italy. ${ }^{6}$ Division of Neonatology and Neonatal Intensive Care, Casilino General Hospital, Roma, Italy.

Received: 21 July 2015 Accepted: 9 December 2015

Published online: 18 December 2015

\section{References}

1. Wahren-Herlenius M, Dörner T. Immunopathogenic mechanisms of systemic autoimmune disease. Lancet. 2013;382:819-31.

2. Cooper GS, Stroehla BC. The epidemiology of autoimmune diseases. Autoimmun Rev. 2003;2:119-25.

3. Ngo ST, Stein FJ, McCombe PA. Gender differences in autoimmune disease. Front Neuroendocrinol. 2014;35:347-69.

4. Østensen M, Khamashta M, Lockshin M, Parke A, Brucato A, Carp H, et al. Anti-inflammatory and immunosuppressive drugs and reproduction. Arthritis Res Ther. 2006:8:209-28.

5. Palmeira P, Quinello C, Silveira-Lessa AL, Zago CA, Carneiro-Sampaio MC. IgG placental transfer in healthy and pathological pregnancies. Clin Dev Immunol. 2012; doi:10.1155/2012/985646.

6. Brucato A, Cimaz R, Caporali R, Ramoni V, Buyon J. Pregnancy outcomes in patients with autoimmune diseases and anti-Ro/SSA antibodies. Clin Rev Allergy Immunol. 2011;40:27-41.

7. Lin HC, Chen SF, Lin HC, Chen YH. Increased risk of adverse pregnancy outcomes in women with rheumatoid arthritis: a nationwide populationbased study. Ann Rheum Dis. 2010;69:715-7.

8. Hon KL, Leung AKC. Neonatal Lupus Erythematosus. Autoimmune Dis. 2012; doi:10.1155/2012/301274

9. Lee LA. Transient autoimmunity related to maternal autoantibodies: neonatal lupus. Autoimmun Rev. 2005;4:207-13.

10. Bykerk VP, Massarotti EM. The new ACR/EULAR classification criteria for RA: how are the new criteria performing in the clinic? Rheumatology. 2012;5:vi16-20.

11. Kramer MS, Zhang X, Platt RW. Analyzing risks of adverse pregnancy outcomes. Am J Epidemiol. 2014;179:361-7.

12. Jaeggi E, Laskin C, Hamilton R, Kingdom J, Silverman E. The Importance of the Level of Maternal Anti-Ro/SSA Antibodies as a Prognostic Marker of the Development of Cardiac Neonatal Lupus Erythematosus. A Prospective Study of 186 Antibody-Exposed Fetuses and Infants. J Am Coll Cardiol. 2010;55:2778-84. 
13. Salomonsson S, Sonesson SE, Ottosson L, Muhallab S, Olsson T, Sunnerhagen M, et al. Ro/SSA autoantibodies directly bind cardiomyocytes, disturb calcium homeostasis, and mediate congenital heart block. J Exp Med. 2005;201:11-7.

14. Ambrosi A, Salomonsson S, Eliasson H, Zeffer E, Skog A, Dzikaite V, et al. Development of heart block in children of SSA/SSB-autoantibody-positive women is associated with maternal age and displays a season-of-birth pattern. Ann Rheum Dis. 2012;71:334-40.

15. Strandberg L, Salomonsson S, Bremme K, Sonesson SE, Wahren-Herlenius M. Ro52, Ro60 and La lgG autoantibody levels and Ro52 lgG subclass profiles longitudinally throughout pregnancy in congenital heart block risk pregnancies. Lupus. 2006;15:346-53.

16. Fuchs KM, Coustan DR. Immunosuppressant therapy in pregnant organ transplant recipients. Semin Perinatol. 2007;31:363-71.

17. Cimaz R, Meregalli E, Biggioggero M, Borghi O, Tincani A, Motta M, et al. Alterations in the immune system of children from mothers treated with immunosuppressive agents during pregnancy. Toxicol Lett. 2004;149:155-62

18. Tincani A, Rebaioli CB, Frassi M, Taglietti M, Gorla R, Cavazzana I, et al. Pregnancy and autoimmnunity: maternal treatment and maternal disease influence on pregnancy outcome. Autoimmun Rev. 2005;4:423-8.

19. Motta M, Zambelloni C, Rodriguez-Perez C, Angeli A, Lojacono A, Tincani A, et al. Cerebral ultrasound abnormalities in infants born to mothers with autoimmune disease. Arch Dis Child Fetal Neonatal Ed. 2011:96:F355-8.

20. Gerosa M, Meroni PL, Cimaz R. Safety considerations when prescribing immunosuppression medication to pregnant women. Expert Opin Drug Saf. 2014;5:1-9.

21. Mekinian A, Lachassinne E, Nicaise-Roland P, Carbillon L, Motta M, Vicaut E, et al. European registry of babies born to mothers with antiphospholipid syndrome. Ann Rheum Dis. 2013;72:217-22.

22. Spinillo A, Beneventi F, Ramoni V, Caporali R, Locatelli E, Simonetta M, et al. Prevalence and significance of previously undiagnosed rheumatic diseases in pregnancy. Ann Rheum Dis. 2012;71:918-23.

\section{Submit your next manuscript to BioMed Central and we will help you at every step:}

- We accept pre-submission inquiries

- Our selector tool helps you to find the most relevant journal

- We provide round the clock customer support

- Convenient online submission

- Thorough peer review

- Inclusion in PubMed and all major indexing services

- Maximum visibility for your research

Submit your manuscript at www biomedcentral.com/submit

) Biomed Central 